\title{
Estudio de la población de carpincho (Hydrochoerus hydrochaeris) en el área natural protegida "El Morejón", Campana (Buenos Aires)
}

\author{
Tatiana Noya Abad ${ }^{12,1}$; Javier A. Calcagno ${ }^{1} \&$ Valeria Bauni $^{3}$
}

\begin{abstract}
${ }^{1}$ CEBBAD-Departamento de Ciencias Naturales y Antropológicas, Universidad Maimónides. Buenos Aires, Argentina. ${ }^{2}$ CONICET-Universidad de Buenos Aires, Instituto de Quimica Biológica, FCEyN (IQUIBICEN), Laboratorio QB81. Buenos Aires, Argentina. ${ }^{3}$ Fundación de Historia Natural Félix de Azara, Universidad Maimónides. Buenos Aires, Argentina.

Resumen. En la Argentina, la Ecorregión Pampeana presenta bajo nivel de protección debido a su alto valor económico y al dominio privado. El reemplazo de sus ambientes naturales por usos productivos causó la retracción numérica de especies como el carpincho (Hydrochoerus hydrochaeris), que habita sus humedales. Los aspectos biológicos y ecológicos de esta especie permanecen poco estudiados en la provincia de Buenos Aires. En consecuencia, los objetivos de este estudio fueron estimar las abundancias real y relativa, la densidad y el tamaño poblacional, y determinar la estructura de la población, el patrón de actividad de los individuos en las dos lagunas presentes en el área natural protegida "El Morejón", partido de Campana, Buenos Aires. Para ello, a lo largo del año 2014 se realizaron relevamientos de transectas de rastros y trampas cámara en las cuatro estaciones. A pesar de estar inmersa en un área urbanizada e industrializada, se obtuvo un tamaño poblacional mayor al esperado. Se estimó la presencia de un promedio de 15 individuos en la Laguna 1 y 154 en la 2, en áreas de uso estimadas de 3 y 10 ha, respectivamente. Durante el invierno se registró el mayor número de individuos, y en otoño, primavera y verano, los menores. Es posible que esto se asocie a la movilidad de los individuos en busca de alimento. Estos resultados son relevantes para una planificación correcta de medidas de manejo, y confirman la importancia de que existan reservas privadas que conserven el ecosistema natural de la región.
\end{abstract}

[Palabras clave: tamaño poblacional, estructura poblacional, transectas de rastros, trampas cámara, estacionalidad, reserva privada]

\begin{abstract}
Study of the population of capybara (Hydrochoerus hydrochaeris) in the protected area "El Morejón", Campana (Buenos Aires). In Argentina, the Pampa ecoregion has a low level of protection due to its high economic value and private domain. The replacement of its natural environments by productive uses has caused the numerical retraction of species such as the capybara (Hydrochoerus hydrochaeris), which inhabits its wetlands. The biological and ecological aspects of this species have been scarcely studied in the province of Buenos Aires. Therefore, the objectives of this study were to estimate the relative, real abundance, density, population size; and determine the structure of the population, the pattern of activity of the individuals in the two lagoons present in the protected natural area "El Morejón", Campana, Buenos Aires. For this purpose, transects of traces and camera traps were made in the four seasons, throughout 2014. Despite being immersed in an urbanized and industrialized area, a larger population size than expected was obtained. An average of 15 individuals in Laguna 1 and 154 in 2 was estimated, in estimated areas of use of 3 and 10 ha, respectively. The largest number of individuals was observed during winter, while fall, spring and summer showed the smallest. The differences may be associated with the alimentary activity. These results are relevant for a correct planning of management measures, and confirm the importance of the existence of private reserves that conserve the natural ecosystem of the region.
\end{abstract}

[Keywords: population size, population structure, transects of traces, camera traps, seasonality, private reserve]

Editor asociado: Alejandro Farii-Brener
Recibido: 28 de Julio de 2019

Aceptado: 11 de Febrero de 2020 


\section{INTRODUCCIÓN}

El carpincho (Hydrochoerus hydrochaeris, suborden Caviomorpha, superfamilia Cavioidea, familia Hydrochoeridae) es el roedor viviente más grande del mundo. Endémico del neotrópico, su distribución está asociada a la presencia de humedales tropicales y subtropicales de Sudamérica. Está presente en todos los países sudamericanos a excepción de Chile (Mones and Ojasti 1986). Se caracteriza por tener tres requerimientos indispensables para su supervivencia: a) la disponibilidad de ambientes acuáticos, b) la presencia de áreas de forrajeo en las cercanías y c) áreas secas para descanso y abrigo de las crías. Mientras que el agua es el requerimiento clave que condiciona la calidad del hábitat del carpincho (i.e., reproducción, termorregulación y dispersión entre diferentes parches), la vegetación es el segundo factor relevante, puesto que influye sobre otros requerimientos básicos (i.e., forraje, sitios de descanso y refugio). A su vez, el tipo y la disposición espacial de los cuerpos de agua en una región condicionará la distribución de las poblaciones de carpinchos en el área (Ojasti and Sosa Burgos 1985; Quintana 1999). Son animales principalmente crepusculares, pero en las zonas donde son disturbados por las actividades humanas, como la que nos ocupa en el presente trabajo, adoptan un estilo de vida nocturno (Ojasti 1973; MacDonald 1981). Viven en grupos muy estables que pueden estar constituidos por un macho cada dos hembras y sus crías, o por un grupo más grande con machos subordinados. El tamaño de los grupos varía entre seis y 20 animales, según la estación del año y el hábitat. Cada grupo está liderado por un macho dominante (Herrera and Macdonald 1987). Durante el verano, cuando hay gran disponibilidad de agua y vegetación, se dispersan (formando grupos más pequeños) y acumulan reservas energéticas en forma de grasa; esto coincide con la cría de los recién nacidos (Ojasti 1973). Por otro lado, durante el invierno, los grupos son más numerosos y la mortalidad es mayor debido a la escases de alimento, a las enfermedades y a la desaparición de las plantas que les sirven de refugio; así se vuelven más vulnerables a los ataques de cazadores y depredadores (Ojasti 1973). La desaparición de los grandes depredadores en muchas áreas coloca al ser humano como la principal amenaza para el carpincho (Schivo et al. 2010). A nivel mundial, la especie está clasificada como de "preocupación menor" (Reid 2016). Sin embargo, a lo largo del tiempo, el riesgo de amenaza de la especie en nuestro país fue en aumento, ya que - según el Libro Rojo de Mamíferos Amenazados de la Argentina (Ojeda et al. 2012) -, el carpincho se encuentra "casi amenazado" a nivel nacional.

En la Reserva Privada "El Morejón", el carpincho parece ser una especie abundante. Habita en las dos lagunas e islotes. Los aspectos relacionados con la biología y ecología de estos animales permanecen poco estudiados en el medio silvestre argentino, por lo que existe un vacío de información, en particular en la provincia de Buenos Aires (Bolkovic and Ramadori 2006). En este contexto, los objetivos de este trabajo fueron estimar la abundancia relativa, la abundancia real, el tamaño poblacional, la densidad de carpinchos, la estructura de edades, las variaciones espacio-temporales de estos parámetros en las dos lagunas, a partir de dos metodologías complementarias: transectas de rastros (método indirecto) y trampas cámara (método directo).

\section{Materiales y MÉTOdos}

La Reserva Privada "El Morejón" se encuentra en la Provincia de Buenos Aires, Argentina $\left(34^{\circ} 11^{\prime} 50^{\prime \prime} \mathrm{S}-59^{\circ} 00^{\prime} 15^{\prime \prime} \mathrm{O}\right.$ ) (Figura 1). "El Morejón" conserva parte de la flora y la fauna característica de la Región Pampeana. Se creó en el 2012 mediante el Programa de Reservas Privadas de la Fundación de Historia Natural Félix de Azara. Ocupa una superficie efectiva de 135.5 ha y la atraviesan dos arroyos: Morejón y La Cruz. El arroyo Morejón posee un cauce poco definido y de escasa profundidad que desemboca en el arroyo La Cruz, y éste, en el río Paraná de las Palmas. A su vez, presenta dos cuerpos de agua artificiales, las lagunas Morejón 1 y 2 (Figura 1), que se formaron hace más de dos décadas como consecuencia de la actividad de extracción de tosca, práctica común en la provincia de Buenos Aires. La Laguna Morejón 1 se ubica próxima a la zona de instalaciones de la Termoeléctrica Manuel Belgrano S.A. (TMB) y presenta una superficie aproximada de 7 ha, con un islote en el medio. La Laguna Morejón 2, a $1.5 \mathrm{~km}$ de las instalaciones de la central eléctrica, abarca $\sim 30$ ha y su configuración más irregular genera la aparición de numerosos islotes de distinto tamaño (Homberg et al. 2014). 

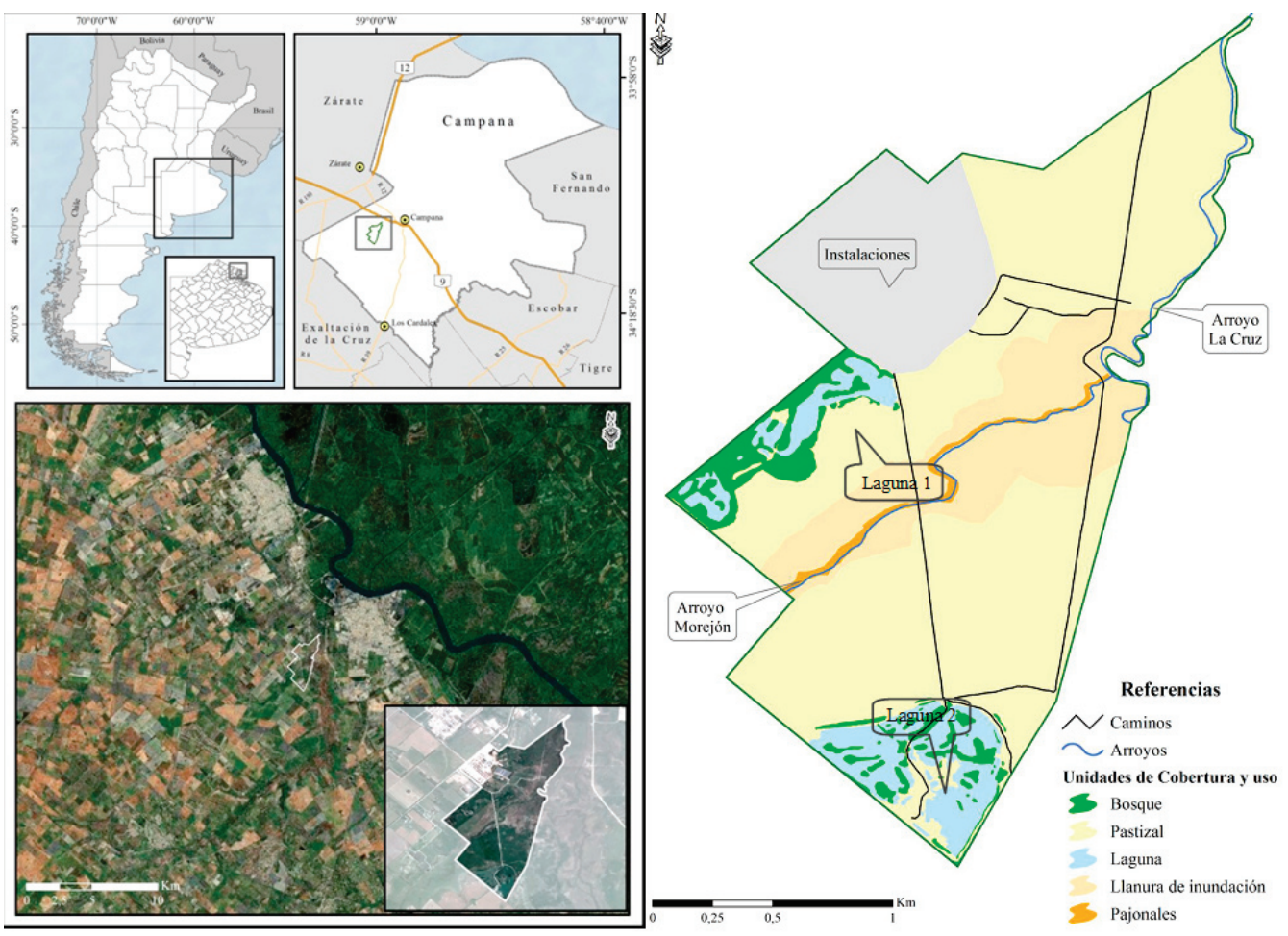

Figura 1. Ubicación de la reserva "El Morejón" y unidades de cobertura del predio Termoeléctrica Manuel Belgrano Figure 1. Location of the "El Morejón" reserve and coverage units of the "Termoeléctrica Manuel Belgrano" property.

\section{Relevamiento a campo}

Debido a que los carpinchos en la reserva son sensibles a la presencia humana, se utilizaron dos metodologías diferentes, una directa y otra indirecta. Ambas son complementarias entre sí: el método indirecto de transectas de indicios basado en el conteo de huellas y heces, y el directo de trampas cámara.

Transectas de rastros. Se realizaron cinco transectas de $100 \mathrm{~m}$ de largo y $3 \mathrm{~m}$ de ancho sobre los senderos que bordean las lagunas 1 y 2, separadas entre sí (como mínimo) por su misma longitud para asegurar la independencia entre las mismas. El punto de inicio de las transectas se determinó al azar mediante el uso de imágenes satelitales. Al transitarlas, se registraron las heces y huellas y se marcaron con el fin de no volver a contarlas nuevamente al día siguiente, evitando la sobreestimación de indicios. Para ello, también se tuvo en cuenta que cada individuo mantiene la misma forma y tamaño de heces por cinco días, observándolas y midiéndolas para no registrar más de una vez la del mismo individuo (comunicación personal M. L. Bolkovic). No se tuvieron en cuenta los rastros de los días previos al inicio del relevamiento, sacándolos de los senderos o marcándolos según fuera conveniente. Los relevamientos se llevaron a cabo durante cinco días en los meses de marzo (verano), mayo (otoño), agosto (invierno) y noviembre (primavera) del año 2014. La posición de las transectas e indicios fueron registradas por medio del uso de un GPS. Se midió cada huella registrada con el fin de obtener información adicional sobre la composición de la población y edad de sus integrantes.

Trampas cámara. Se colocaron seis cámaras fotográficas MOULTRIE M-80XT, tres en cada laguna. Estas cámaras poseen un sensor infrarrojo que activa la toma de la fotografía en forma automática al detectar el movimiento y calor generado por un animal u objeto dentro de un área conocida como "zona de sensibilidad" (Chávez et al. 2013). Las cámaras se sujetaron a árboles o arbustos a $40 \mathrm{~cm}$ del suelo y se despejó la vegetación frente a las mismas para que no dispararan continuamente, con la precaución de modificar el sitio lo menos posible para que los animales no percibieran diferencias. Se aseguraron firmemente para evitar que el viento cambie su orientación, y se posicionaron de forma 
tal que el sensor tenga suficiente campo libre para captar la presencia del animal. Para obtener fotografías enfocadas y de animales completos, se intentó colocar las cámaras a una distancia superior a $1.5 \mathrm{~m}$ del lugar donde se espera que pase el animal, tal como se realizó en el trabajo de Perovic et al. (2008). Al día siguiente de la instalación de las estaciones de foto-trampeo se verificó la correcta posición de la cámara. Para la ubicación de las cámaras, en cada laguna se seleccionaron tres lugares (una cámara por lugar) a $500 \mathrm{~m}$ de distancia uno del otro, donde anteriormente se observaron rastros, abarcando la mayor parte posible del perímetro de las lagunas y asegurando la independencia entre las estaciones de foto trampeo. Cada estación fue georreferenciada con un GPS. El esfuerzo de muestreo fue de cuatro días para cada estación del año 2014. La misma metodología se repitió durante las cuatro estaciones del año, desde el verano a la primavera del 2014.

\section{Análisis de datos}

Transectas de rastros. Con el número de indicios obtenido a través del método de transectas se estimó la abundancia relativa (Carrillo et al. 2000):

$\mathrm{IAR}_{\mathrm{T}}=($ nro. de indicios / unidad de esfuerzo $)$ $\times 100$

(Ecuación 1)

La abundancia real (AR), la densidad de individuos y el tamaño poblacional se estimaron utilizando la tasa de defecación diaria (TDD) promedio de 4.4 heces/día calculada en una laguna de una estancia de Corrientes (Bolkovic et al. 2006):

$$
\mathrm{AR}=\mathrm{IAR}_{\text {heces }} / \mathrm{TDD}
$$

(Ecuación 2)

Densidad de individuos $=$ (nro. de deposiciones promedio/TDD) / unidad de esfuerzo

(Ecuación 3)

Tamaño poblacional $=$ densidad deindividuos $x$ área estimada de uso

(Ecuación 4)

Para evaluar diferencias entre los factores analizados se realizó un modelo mixto lineal generalizado (GLMM) con distribución de Poisson, utilizando pruebas secuenciales de los efectos fijos simples y comparaciones múltiples DGC. Se consideraron los efectos de las lagunas, las estaciones e interacción entre ambas. Todos los análisis estadísticos descriptos fueron realizados utilizando el programa Infostat (Di Rienzo et al. 2009).
Para estimar la edad de los individuos registrados a través de las huellas se utilizó una relación basada en el estudio del crecimiento de las crías de grupos silvestres y de criaderos desarrollada por Soini (1996). La misma se basa en la relación aproximada entre la edad y la longitud total de la huella de las patas traseras que se diferencian de las delanteras al tener tres dedos en vez de cuatro.

Trampas cámara. Para analizar los registros obtenidos a través de las trampas cámara se calculó el porcentaje de fotos efectivas (Lozano 2010): $\%$ fotos efectivas $=($ nro. de fotos efectivas $/$
nro. total de fotos $) \times 100 \quad$ (Ecuación 5$)$

El índice de abundancia relativa $\left(\mathrm{IAR}_{\mathrm{TC}}\right)$ para los datos fotográficos se calculó como:

$\mathrm{IAR}_{\mathrm{TC}}=(\mathrm{C} / \mathrm{EM}) \times$ unidad estándar

(Ecuación 6)

donde $\mathrm{C}$ son las capturas del individuo de interés y EM es el esfuerzo de muestreo (nro. de cámaras x días de muestreo, es decir 3 x 4) (Azuara 2005; Jenks et al. 2011).

En cada fotografía queda registrada la hora en el que se efectuó el disparo. Mediante esta información, se analizó el patrón de actividad de los carpinchos. Se agruparon bandas horarias de cuatro horas a las cuales se le asignaron los números de registros de carpinchos. A partir de las mismas se calcularon los patrones de actividad para cada estación y para el año relevado completo.

\section{Resultados}

\section{Parámetros poblacionales}

Transectas de rastros. En los relevamientos realizados en la reserva "El Morejón" durante el año 2014 se recorrieron 18000 metros. En los mismos se registraron 1463 rastros de carpinchos, de los cuales el 85\% corresponde a heces y el $15 \%$ a huellas.

El índice de abundancia relativa, la abundancia real, densidad y tamaño poblacional fueron superiores en la Laguna 2, en las cuatro estaciones analizadas. Asimismo, en invierno se obtuvieron los valores más altos de dichos parámetros, seguido del otoño, luego primavera y por último verano (Tabla 1). Se estima una población promedio anual aproximada de 15 individuos en la Laguna 1, y de 154 en la Laguna 2. Se aplicó un GLMM 
Tabla 1. Índice de abundancia relativa (IART), abundancia real (ART) y densidad y tamaño poblacional (N), según el método de transectas. Letras distintas indican diferencias significativas $(P<0.05)$.

Table 1. Index of relative abundance (IRA), real abundance (RA), density and population size (N), according to the transect method. Different letters indicate significant differences $(P<0.05)$.

\begin{tabular}{clccccccc}
\hline Laguna & Estación & $\begin{array}{c}\text { IART } \\
\text { (indicios/100 } \mathrm{m} \text { ) }\end{array}$ & $\begin{array}{c}\text { IART } \\
\text { (heces/100 m) }\end{array}$ & ART & $\begin{array}{c}\text { Densidad } \\
\text { (ind/ha) }\end{array}$ & $\begin{array}{c}\mathrm{N} \\
\text { (ind) }\end{array}$ & $\begin{array}{c}\text { DGC } \\
\text { Laguna }\end{array}$ & $\begin{array}{c}\text { DGC } \\
\text { Estación }\end{array}$ \\
\hline \multirow{4}{*}{ L1 } & Verano & 0.22 & 0.22 & 0.05 & 1.67 & 5.00 & & $\mathrm{~A}$ \\
& Otoño & 0.58 & 0.53 & 0.12 & 4.00 & 12.00 & & $\mathrm{~A}$ \\
& Invierno & 1.34 & 1.27 & 0.29 & 9.64 & 28.91 & $\mathrm{~A}$ & $\mathrm{~B}$ \\
& Primavera & 0.63 & 0.55 & 0.13 & 4.17 & 12.50 & & $\mathrm{~A}$ \\
& Verano & 1.46 & 1.29 & 0.29 & 9.77 & 97.73 & $\mathrm{~A}$ \\
L2 & Otoño & 2.71 & 2.17 & 0.49 & 16.42 & 164.24 & & $\mathrm{~A}$ \\
& Invierno & 3.97 & 3.25 & 0.74 & 24.61 & 246.06 & $\mathrm{~B}$ & $\mathrm{~B}$ \\
& Primavera & 1.53 & 1.38 & 0.31 & 10.45 & 104.55 & & $\mathrm{~A}$ \\
\hline
\end{tabular}

con distribución de Poisson. Se realizaron comparaciones múltiples DGC mediante pruebas de hipótesis secuenciales para los efectos fijos del modelo. Se detectaron diferencias significativas entre lagunas teniendo en cuenta o no la estación. Por otro lado, sólo la estación invierno resultó significativamente diferente de las demás (Tabla 1). A partir de las huellas traseras identificadas y medidas en las transectas en las cuatro estaciones, se registraron crías, juveniles, sub-adultos, adultos en la Laguna 2 , mientras que en la Laguna 1 solo juveniles, sub-adultos y adultos (Figura 2).

Trampas cámara. Mediante las trampas cámara se obtuvieron un total de 144 fotografías, de las cuales 50.69\% correspondieron a carpinchos. De éstos, $35.62 \%$ fueron en la Laguna 1 y $64.38 \%$ fueron en la Laguna 2. Otras especies registradas a través de este método fueron el gato montés (Leopardus geoffroyi), el zorro gris pampeano (Pseudalopex gymnocercus), la comadreja overa (Didelphis albiventris), el lagarto overo (Salvator merianae) y algunas especies de aves. En la Laguna 1 se obtuvo el mayor porcentaje de fotos efectivas en primavera, mientras que en la Laguna 2 en verano. En ambas, el menor porcentaje fue en invierno.

El índice de abundancia relativa $\left(\mathrm{IAR}_{\mathrm{TC}}\right)$ en la Laguna 1 fue mayor en verano e invierno seguido de primavera y otoño, mientras que en la Laguna 2 lo fue en verano y otoño seguido de primavera e invierno (Tabla 2). El IAR anual promedio estimado fue de 2.29 en la Laguna 1 y de 3.92 en la Laguna 2.

Mediante los datos otorgados por las fotografías obtenidas por medio de las trampas cámara se determinaron rangos de horario con mayor, menor o nula actividad de los carpinchos. En el muestreo de verano el mayor porcentaje de capturas se obtuvo en los rangos de 4:00 a 8:00 h y de 20:00 a 00:00 h, con $30 \%$ y $33.33 \%$, respectivamente. En otoño, se concentró en el rango de 4:00 a 8:00 h, con un $68 \%$. En invierno fue en el rango de 16:00 a $20: 00 \mathrm{~h}$, con $55.56 \%$ y $22.22 \%$, mientras que en primavera fue en el rango de 20:00 a 00:00 h, con un $60 \%$ (Figura 3 ).

Por otro lado, la temperatura mínima a la cual se registró un individuo de carpincho por

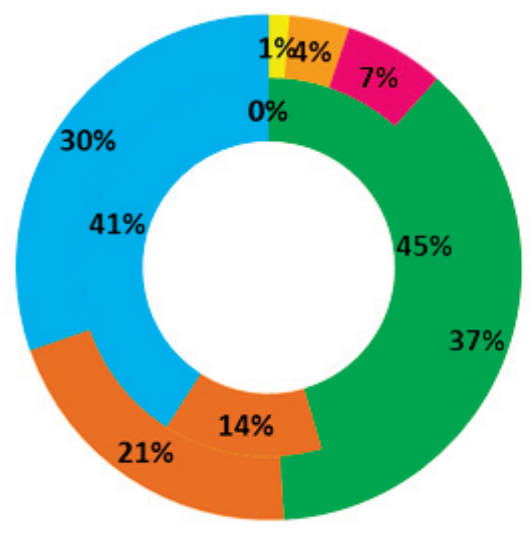

crías menores de un mes

In crías de uno a dos meses

- crías de tres meses o más

E juveniles de seis meses o más

nubadultos de un año de edad

adultos
Figura 2. Porcentaje de las categorías de edad en las lagunas 1 y 2 , según el método de transectas.

Figure 2. Percentage of age categories in lagoons 1 and 2 , according to the transect method. 

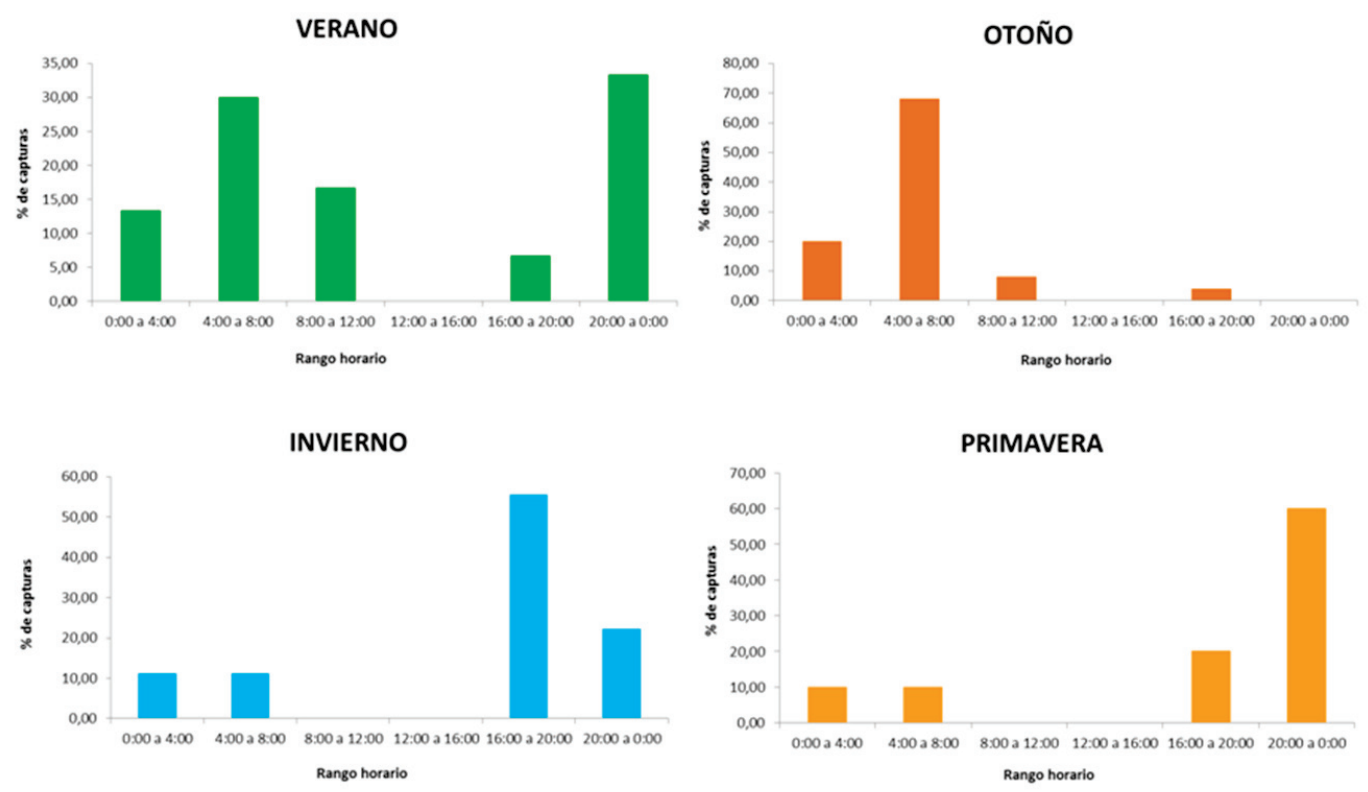

Figura 3. Porcentaje de individuos de carpincho registrados por trampas cámara en cada rango horario en cada estación, teniendo en cuenta las dos lagunas.

Figure 3. Percentage of capybara individuals registered by camera traps in each hourly range in each station, taking into account the two lagoons.

\begin{tabular}{cccccc}
\hline Laguna & Estación & Capturas & $\begin{array}{c}\text { Esfuerzo } \\
\text { de muestreo }\end{array}$ & $\begin{array}{c}\text { Unidad } \\
\text { estándar }\end{array}$ & $\begin{array}{c}\text { IAR }_{\mathrm{TC}} \\
\text { (capturas/EM) }\end{array}$ \\
\hline \multirow{4}{*}{ L1 } & Verano & 8 & 12 & & 2.67 \\
& Otoño & 3 & 8 & 4 & 1.50 \\
& Invierno & 8 & 12 & & 2.67 \\
& Primavera & 8 & 12 & & 2.67 \\
\multirow{4}{*}{ L2 } & Verano & 22 & 12 & & 7.33 \\
& Otoño & 22 & 12 & 4 & 7.33 \\
& Invierno & 1 & 12 & & 0.33 \\
& Primavera & 2 & 12 & & 0.67 \\
\hline
\end{tabular}

Tabla 2. Índice de abundancia relativa (IAR) según el método de trampas-cámara.

Table 2. Index of relative abundance (IRA) according to the camera-trap method.

medio de las trampas cámara fue de $-2^{\circ} \mathrm{C}$ en invierno, y la máxima fue de $26^{\circ} \mathrm{C}$ en verano, ambos en la Laguna 1. Mientras tanto, en la Laguna 2, la mayor temperatura a la cual se capturo fue de $17^{\circ} \mathrm{C}$ en verano y primavera, y la menor fue de $1^{\circ} \mathrm{C}$ en invierno.

\section{DisCUSIÓN}

Este estudio representa la primera estimación de los distintos parámetros de la población de carpinchos realizada en la reserva "El Morejón". Esto es de vital importancia para lograr una correcta evaluación de la población presente y poder realizar planes de conservación y medidas de manejo, ya que estimar la densidad y tamaño poblacional es una información imprescindible para tomar decisiones. Además, aporta al conocimiento sobre esta especie en la provincia de Buenos
Aires, que corresponde con el límite más austral de su distribución.

Las estimaciones de densidad y de tamaño de la población de carpinchos obtenidos a través del conteo de indicios en la reserva fue alto, sobre todo en la Laguna 2, considerando el contexto industrial y urbanizado en el cual se encuentra inmersa; esto evidencia una diferencia significativa de los parámetros poblacionales calculados entre lagunas. Por otro lado, los resultados obtenidos mediante el método de transectas de ancho fijo muestran una diferencia estacional en la población, en particular con un mayor número de individuos en invierno. Al comparar estaciones no se obtuvo el mismo patrón con las trampas cámara. Tanto la diferencia entre estaciones como entre metodologías probablemente se deba a que la forma de los cuerpos de agua 
en el paisaje y la disponibilidad de recursos podría estar condicionando la distribución y el uso del hábitat de los individuos en el área a lo largo del año. Durante el invierno, cuando escasea la vegetación, harían un mayor uso de la reserva y se dispersarían en busca de recursos, lo cual se evidencia a través de un mayor número de indicios en los senderos, pero un menor número de capturas por medio de las trampas cámara, debido a que fueron ubicadas bordeando a las lagunas. En primavera y verano se concentrarían en los islotes presentes en las lagunas o muy cercanas a las mismas, gracias a la abundancia y la diversidad de la vegetación, obteniendo un menor número de indicios en los senderos, pero un mayor número de fotografías en los bordes de las lagunas. Esto coincide con el comportamiento que planteó Ojasti (1973). A su vez, Rimoldi y Chimento (2014) estimaron la abundancia relativa de carpinchos en un bosque xerófito ribereño de la cuenca del río Carcarañá, en la provincia de Santa Fe, en las cuatro estaciones entre el año 2011 y 2012, mediante el método de transectas. En ese trabajo se obtuvieron diferencias significativas entre las mismas y un patrón similar al de nuestro estudio.

Un estudio realizado Rabinovich et al. (2003) en un tajamar (represa de contención de agua de campos arroceros) de la Estancia San Luis de las Mulas (Entre Ríos), en el que se realizó el método de transectas para dos sectores (A y B), se obtuvieron 3.8 capinchos/ha u 8 individuos en un área de 1.9 ha en el sector A, mientras que en el sector B se registraron 11.58 carpinchos/ha ó 12 individuos en un área de 1 ha. Podemos deducir que lo obtenido en nuestro trabajo es similar (siendo proporcional el tamaño del área disponible para la especie y el número de individuos presentes) dado que estimamos un promedio anual de 4.87 carpinchos/ha ó 15 individuos en un área de 3 ha (áreas de uso estimada de la Laguna 1), y 15.31 capinchos/ha ó 154 individuos en un área de 10 ha (área de uso estimada de la Laguna 2).

El territorio de los grupos de carpinchos fue estimado en $~ 10$ ha para áreas con altas densidades poblacionales (De Azcárate 1981; MacDonald 1981). La lagunas 1 y la 2 poseen 3 y 10 ha de islotes y senderos que las rodean, respectivamente, y cubren la superficie necesaria para la especie. Sin embargo, según De Azcárate (1980) transcurren la mayor parte del tiempo en áreas centrales (core áreas) de menos de 1 ha, semejante al tamaño que tienen los islotes presentes en las lagunas. Por ende, es posible que los carpinchos de la Laguna 1 no se dirijan a la 2 ni viceversa, lo que explicaría la falta de indicios evidenciada en el pastizal, llanura de inundación y pajonal, al realizar el muestreo piloto.

Los datos obtenidos mediante los distintos métodos desarrollados evidencian una diferencia marcada en la abundancia de carpinchos entre ambas lagunas. Varios estudios (Bolkovic et al. 2011; Rimoldi and Chimento 2014) muestran que la cercanía a áreas urbanas, industrializadas, con actividad humana, donde existe una mayor presión de caza, estos valores disminuyen; esto podría estar sucediendo en esta reserva. A su vez, la presencia de mayor vegetación e islotes dificultan más el acceso de las personas a la Laguna 2 que a la 1. Según Ojasti (1978), el número de carpinchos en el lugar depende del tamaño de los cuerpos de agua, de la presencia de zonas de pastura y la cobertura vegetal del lugar; es decir, la calidad del hábitat. Por ende, sitios con una alta idoneidad del hábitat presentan mayor densidad respecto a otros con baja calidad, respecto a la disponibilidad de forraje. Pero si los primeros presentan una alta presión de caza, se invierte. La mayor disponibilidad agua, de vegetación, de sitios inaccesibles para el humano y de menor presión de caza permiten una mayor densidad de carpincho (Quintana 1996), tal como se observa con la Laguna 2 de nuestra área de estudio. Según la estructura de edad resultante, a diferencia de la Laguna 2, la falta de registros de crías en la Laguna 1 sugiere que los individuos no reprodujeron en todo el año, en relación con una menor disponibilidad de buenos sitios de descanso y refugio, mayor presión de caza, o debido a que allí se encuentran los machos subordinados acompañados de subadultos (tal como suele ocurrir según Herrera [1999]). De esta manera se puede inferir que la causa de un aumento en los valores de los distintos parámetros en la Laguna 2 podría deberse a su mayor superficie, número de islotes, cobertura vegetal, disponibilidad de recursos, menor presión de caza, mayor distancia a la Ruta Nacional 9 e instalaciones de la central eléctrica y de la empresa lindera a la termoeléctrica; dicho sector de la reserva les resulta más viable a los carpinchos. Si bien el porcentaje de crías evidenciado por las 
huellas fue bajo, mediante observación directa y las trampas cámara fueron observadas con frecuencia.

Por otro lado, mediante el método de trampas cámara no se estimó la densidad poblacional, ya que sólo se puede estimar para especies con marcas individuales. En este trabajo, las trampas cámara complementan el estudio y proveen información adicional a la que se puede conseguir con la observación de heces y de huellas, obteniendo datos confiables, completos y patrones complementarios con ambos métodos. Según el patrón de actividad logrado mediante el análisis de las trampas cámara, los carpinchos de la reserva son principalmente crepusculares y tienen un porcentaje considerable de actividad nocturna, posiblemente debido a que no tienen un alto nivel de depredación, pero sí son molestados por las actividades humanas y son cazados de forma furtiva.

Para estimar la densidad y el tamaño poblacional de carpinchos, las transectas de indicios parecen ser el método más efectivo, simple, económico y rápido, ya que permite estimar parámetros poblacionales. Sin embargo, usar metodologías complementarias permite recopilar información más completa sobre el objeto de estudio, tal como plantean los informes de Bolkovic et al. (2007) y Bolkovic (2013) para la Dirección de Fauna Silvestre.

El cambio en el uso del suelo es cada vez más intenso en los pastizales pampeanos, y estas modificaciones en la estructura y la composición del paisaje afectan de manera directa a los mamíferos locales (Homberg et al. 2014). Para conservar de manera efectiva la diversidad biológica requiere conocer los componentes o los elementos que la conforman. En la Reserva Privada "El Morejón", los carpinchos son la única especie de mamífero con algún grado de amenaza. La Reserva abarca ambientes de pastizal, bosque ribereño y humedales, que en el contexto ambiental de la Ecorregión Pampeana constituyen ambientes de alto valor para la conservación y brinda hábitat propicio para la especie estudiada.

A partir de los resultados del presente trabajo pudimos obtener información básica sobre esta población, que, a pesar de estar inmersa en un área urbanizada e industrializada, alberga un importante número de individuos, posiblemente porque es el único sitio en la zona con hábitat propicio (i.e., disponibilidad de recursos y presencia de cuerpos de agua).

Los resultados son de importancia vital para una planificación correcta de medidas de manejo, confirman cuán relevante es que existan reservas privadas que conserven el ecosistema natural de la región y refuerzan la existencia de "El Morejón". A su vez, tal como dice Bolkovic et al. (2007), el carpincho muestra un amplio potencial biológico para ser manejado en el medio natural. Esta característica, sumada al hecho de su importancia comercial como producto de fauna silvestre, lo coloca en una situación que requiere establecer una estrategia común de gestión que incluya tanto a la Nación como a todas las provincias y lugares con poblaciones de esta especie. Estudiar las poblaciones presentes permitirá no sólo implementar un plan de uso sustentable para la especie, sino, a su vez, llevar a cabo acciones tendientes a conservar los humedales. A su vez, contar con un manejo sustentable redundará también en un beneficio para otras especies asociadas a los hábitats del carpincho y para las comunidades humanas relacionadas con su explotación o uso de su ambiente.

Agradecimientos. A la Fundación de Historia Natural Félix de Azara, la Termoeléctrica Manuel Belgrano S.A. y la Universidad Maimónides por el financiamiento y apoyo logístico. Gracias a Sebastián Sabatini y Javier Calcagno por incentivarme a escribir este trabajo.

\section{REFERENCIAS}

Azuara, S. D. 2005. Estimación de abundancia de mamíferos terrestres en un área de la Selva Lacandona, Chiapas. Tesis Licenciatura. Facultad de Ciencias, UNAM.

Bolkovic, M. L. 2013 Análisis de métodos de estimación de abundancias y monitoreo de carpinchos (Hydrochoerus hydrochaeris) para la Argentina. Informe técnico. Dirección de fauna silvestre, SAyDS, Argentina. Pp. 14.

Bolkovic, M. L., R. C. D’Angelo, and M. A. T. Eberhardt. 2011. Evaluación de presencia de carpinchos (Hydrochoerus hydrochaeris) en dos arroyos con diferente presión de caza en islas del Paraná Medio, provincia de Santa Fe. XXIV Jornada Argentina de Mastozoología. SAREM. La Plata, Buenos Aires.

Bolkovic, M. L., R. D. Quintana, D. E. Ramadori, M. Elisetch, and J. Rabinovich. 2007. Proyecto carpincho. Manejo de Fauna en la Argentina: Programas de Uso Sustentable. Informe técnico. Dirección de fauna silvestre, SAyDS, Argentina. Pp. 15. 
Bolkovic, M. L., and D. Ramadori (eds.). 2006. Manejo de Fauna Silvestre en la Argentina. Programas de uso sustentable". Dirección de Fauna Silvestre, Secretaría de Ambiente y Desarrollo Sustentable, Buenos Aires. Pp. 168.

Carrillo, E., G. Wong, and A. Cuarón. 2000. Monitoring mammal populations in Costa Rican protected areas under different hunting restriction. Conservation Biology 14(6):1580-1591. https://doi.org/10.1111/j.1523-1739.2000.99103.x. https:/ / doi.org/10.1046/j.1523-1739.2000.99103.x.

Chávez, C., A. de la Torre, H. Bárcenas, R. A. Medellín, H. Zarza, and G. Ceballos. 2013. Manual de fototrampeo para estudio de fauna silvestre. El jaguar en México como estudio de caso. Alianza WWF-Telcel. Universidad Nacional Autónoma de México, México. Pp. 108.

Cordero, G., and J. Ojasti. 1981. Comparison of capybara populations of open and forested habitats. J Wildl Manage 45(1):267-271. https://doi.org/10.2307/3807900.

De Azcárate, T. 1981. Sociobiologia y manejo del chiguire. Doñana Acta Vertebrata 7:1-228.

Di Rienzo, J. A., F. Casanoves, M. G. Balzarini, L. González, M. Tablada, and C. W. Robledo. 2009. InfoStat. Grupo Infostat, Facultad de Ciencias Agropecuarias, Universidad Nacional de Córdoba. Argentina.

Herrera, E. A. 1999. Comportamiento, conservación y manejo de fauna silvestre: el caso del capybara en Venezuela. Etología 7:41-46.

Herrera, E. A., and D. W. Macdonald. 1987. Group stability and the structure of a capybara population. Symp. Zool. Soc. London 58:115-130.

Homberg, M. A., V. Bauni, S. Bogan, and J. M. Meluso. 2014. Plan de Manejo Ambiental de la Reserva Natural Privada "El Morejón". Pp. 139.

Jenks, K. E., P. Chanteap, K. Damrongchainarong, P. Cutter, T. Redford, A. J. Lynam, J. Howard, and P. Leimgruber. 2011. Using Relative Abundance Indices from Camera-Trapping to Test Wildlife Conservation Hypotheses - An Example from Khao Yai National Park, Thailand. Tropical Conservation Science 4:113-131. https: / /doi.org/10.1177/ 194008291100400203.

Lozano, R. L. A. 2010. Abundancia relativa y distribución de mamíferos medianos y grandes en dos coberturas vegetales en el santuario de fauna y flora Otún Quimbaya mediante el uso de cámaras trampa. Tesis, carrera de Biología. Pontificia Universidad Javeriana, Facultad de ciencias. Bogotá. D.C. Pp. 43.

MacDonald, D. 1981. Dwindling resources and the social behavior of capybaras (Hydrochoerus hydrochaeris) (Mammalia). J Zool London 194:371-391. https://doi.org/10.1111/j.1469-7998.1981.tb04588.x.

Mones, A., and J. Ojasti. 1986. Hydrochoerus hydrochaeris. The American Society of Mammalogists. Mammalian Species 264:7. https://doi.org/10.2307/3503784.

Ojasti, J. 1973. Estudio biológico del chigüire o capibara. Fondo Nacional de Investigaciones Agropecuarias. Caracas. Pp. 275.

Ojasti, J. 1978. The relation between popa Won and production of the capybara. Ph.D. Thesis. Univ. Georgia, Athens, Greece.

Ojasti, J., and L. Sosa Burgos. 1985. Density regulation in the populations of capybara. Acta Zoologica Fennica 173: 81-83.

Ojeda, R. A., V. Chillo, and G. B. Diaz Isenrath (eds.). 2012. Libro Rojo de Mamíferos Amenazados de la Argentina. SAREM. Buenos Aires. Pp. 257.

Perovic, P., C. Trucco, A. Tálamo, V. Quiroga, D. Ramallo, A. Lacci, A. Baungardner, and F. Mohr. 2008. Guía técnica para el monitoreo de la biodiversidad. Programa de Monitoreo de Biodiversidad - Parque Nacional Copo, Parque y Reserva Provincial Copo, y Zona de Amortiguamiento. APN/GEF/BIRF. Salta, Argentina. Pp. 74.

Quintana, R. D. 1996. Análisis y evaluación de la aptitud de hábitat del carpincho (Hydrochoeris hydrohaeris) en relación con la heterogeneidad del paisaje y las interacciones con ganado doméstico. Tesis doctoral, Universidad de Buenos Aires. Pp. 270.

Quintana, R. D. 1999. Relationship between a wetland landscape structure and wildlife: the capybara (Hydrochoeris hydrochaeris) as a study case. Pp. 185-204 en A. I. Malvárez (ed.). Tópicos sobre humedales subtropicales y templados de Sudamérica. ORCyT - MAB/UNESCO, Montevideo. Buenos Aires. Pp. 81.

Quintana, R. D., and J. Rabinovich. 1993. Assessment of capybara (Hydrochoeris hydrochaeris) populations in the wetlands of Corrientes, Argentina. Wetlands Ecology and Management 2:223-230. https://doi.org/10.1007/BF00188156.

Rabinovich, J. E., D. E. Ramadori, M. Elisetch, M. L. Bolkovic, A. Malpartida, and R. D. Quintana. 2003. Proyecto carpincho: Desarrollo de un plan de manejo sustentable de poblaciones silvestres de carpinchos (Hydrochoeris hydrochaeris).

Reid, F. 2016. Hydrochoerus hydrochaeris. The IUCN Red List of Threatened Species 2016. URL: tinyurl.com/y86fyjno.

Rimoldi, P. G., and N. R. Chimento. 2014. Presencia de Hydrochoerus hydrochaeris (Hydrochoeridae, Rodentia, Mammalia) en la cuenca del río Carcarañá, Santa Fe, Argentina. Comentarios acerca de su conservación y biogeografía en Argentina. Pap Avulsos Zool 54(23):329-340. São Paulo. https:/ / doi.org/10.1590/0031-1049.2014.54.23.

Schivo, F., P. Kandus, P. Menotti, and R. D. Quintana. 2010. Mapa de aptitud ecológica potencial para el carpincho (Hydrochoeris hydrochaeris) en la provincia de Corrientes, Argentina. Asociación Argentina de Ecología de Paisajes. RASADEP 1:83-100.

Soini, P. 1996. Un estudio de la abundancia del ronsoco (Hydrochaeris hydrochaeris) en la Reserva Nacional Pacaya Samiria y propuesta de un método de censo. Folia Amazónica 8(1):163-176. https://doi.org/10.24841/fa.v8i1.312.

Soule, M., and K. Kohm. 1989. Research Priorities for Conservation Biology. Island Press, Washington D.C. Pp. 95. 